\title{
POLÍTICAS DE GESTIÓN DEL CUERPO ACADÉMICO EN LA PONTIFICIA UNIVERSIDAD CATÓLICA DE CHILE
}

\section{Introducción}

La Pontificia Universidad Católica de Chile (PUC), en sus 120 años de existencia, ha evolucionado en su esencia y concepción. Desde sus inicios hasta la primera mitad del siglo pasado fue una universidad principalmente focalizada hacia la docencia; en los años siguientes privilegió la investigación; hoy es una institución de cara a la sociedad y abierta a la innovación. En la PUC se enseña lo que se investiga; de este modo, se busca que los académicos divulguen sus conocimientos en las aulas. Este paradigma expresa la voluntad de que su cuerpo académico genere un ambiente educativo estimulante por la presentación actualizada, crítica e incisiva de los conocimientos disponibles en un determinado campo disciplinario.

Sin embargo, docencia e investigación no son las únicas actividades desarrolladas por sus profesores, quienes, a su vez, realizan actividades de extensión y gestión académica. Hasta el siglo XX, el paradigma habitual en las universidades fue el de académicos con capacidad docente que fueran, a la vez, buenos investigadores y que mostraran disposición y capacidad para ejecutar labores de extensión y/o de gestión, aunque estas últimas no fueran necesariamente consideradas en el currículo del profesor y, consecuentemente, no aportaran a su avance en la carrera académica. Es decir, ella estaba trazada principalmente sobre un molde común, de escasa flexibilidad, al cual los académicos debían adaptarse. Por lo tanto, un modelo de este tipo dejaba en cierta desventaja a unidades vinculadas a las artes o a sectores profesionales en los que la investigación básica era menos evidente. 
Por otra parte, una universidad compleja como la PUC debe responder a un Plan de Desarrollo Institucional diverso y desafiante, alineando para ello a sus distintas unidades en torno a metas comunes que puedan concretarse. Por lo mismo, los académicos tienen un papel fundamental en llevar a cabo las diferentes tareas asignadas a cada unidad.

En este trabajo se describe la forma en que la PUC se ha propuesto enfrentar el desafío de responder a su Plan de Desarrollo Institucional y a los planes estratégicos de las unidades, a través de una nueva Política de Gestión del Cuerpo Académico, plasmada en un reglamento, de reciente aprobación, que permita la diversidad de vocaciones e intereses y, por lo tanto, distintas posibilidades de avanzar en la carrera académica. Asimismo, que reconozca que actividades como gestión y extensión también constituyen acciones de notabilidad para la institución y, consiguientemente, para la carrera académica de los profesores, y que contemple un sistema de remuneraciones vinculado al desempeño del académico.

El esquema planteado anteriormente, que constituiría la base del modelo de desarrollo institucional para los próximos años, contiene diversos grados de progreso de las temáticas descritas. Por una parte, la política de calificación formal, el sistema de remuneraciones y los programas de acogida ya son parte de la cultura y modelo de gestión institucional, mientras que aspectos como carrera académica diferenciada, procesos de selección con estándares homogéneos mínimos para todas las unidades académicas y evaluación por perfiles se encuentran en una etapa incipiente de desarrollo.

\section{Una nueva política de gestión}

En gran medida, las instituciones son el reflejo de las personas que las integran y las universidades no son una excepción a esta regla. Aquellas universidades que han alcanzado renombre internacional han mantenido a través de sucesivas generaciones un cuerpo académico de gran calidad, excelencia y comprometidos plenamente con su misión. 
Sin duda, esta capacidad demostrada requiere de adecuadas políticas de gestión del estamento académico, orientadas a lograr que este cuerpo encuentre las condiciones necesarias para el desarrollo de un proyecto de vida académica, en un ambiente de trabajo estimulante y que reconozca y premie el trabajo bien logrado.

El desarrollo del cuerpo académico demanda, por tanto, una continua revisión y perfeccionamiento de sus estructuras y mecanismos, con el fin de avanzar hacia un marco de políticas que se caracterice por su efectividad y eficacia, fundadas en prácticas y valores propios de la institución, base sobre la cual una universidad puede aspirar a un alto nivel de excelencia.

Para avanzar en tal dirección, a inicios de 2008 el Consejo Superior de nuestra universidad aprobó una nueva Política de Gestión del Cuerpo Académico "instrumentalizada en el reglamento correspondiente", que presenta diez lineamientos clave, los cuales se organizan en dos grandes grupos detallados a continuación.

\subsection{Política de Gestión del Cuerpo Académico: conceptos fundamentales}

\subsubsection{Gestión centrada en las personas}

El primer elemento de una adecuada política de gestión académica es el cuidado de las personas. Por ello se entiende la forma en que la autoridad universitaria, en sus diversos niveles, actúa para seleccionar en forma acertada y para acoger, motivar y orientar a los académicos, actuando, a su vez, como guía y facilitadora de un proceso de desarrollo personal y profesional. Esto implica avanzar hacia el logro de una alineación sinérgica entre los planes institucionales y los proyectos individuales de los profesores.

Aunque lo anterior parezca obvio, con mucha frecuencia las instituciones universitarias tienden a una práctica administrativa más centrada en las tareas que en la gente y, por tanto, sus autoridades suelen priorizar la gestión de recursos materiales por sobre la de 
personas. De allí que la adopción de nuevas políticas de gestión académica requiere, en primer término, hacer conscientes a las autoridades de que una de sus principales responsabilidades es la de procurar que cada académico pueda desarrollarse de manera integral, tanto en el plano personal como en el profesional, buscando incorporar sus capacidades y aportes específicos a los desafíos de la respectiva unidad académica y universidad.

Esto requiere claridad respecto de los objetivos institucionales, voluntad, método y perseverancia. Se trata de realizar un trabajo personalizado, con un diálogo sincero y eficaz que aliente las buenas prácticas y enmiende, cuando corresponda, las posibles desviaciones respecto de metas pactadas.

\subsubsection{Fortalecimiento de nuestra identidad institucional}

Toda acción verdaderamente educativa exige un fundamento antropológico, así como todo conocimiento es producto de una razón movida o conmovida por una determinada visión del mundo. Por lo mismo, resulta relevante presentar la impronta que distingue la naturaleza y el fundamento de la institución. De esta manera, los académicos se podrán sentir representados e interpelados por esa marca de origen y misión, ya que la comunicación de un saber lleva consigo la expresión implícita o explícita de esa visión y de los valores que ella establece.

Por otra parte, como se mencionó, en la PUC "se enseña lo que se investiga”. Esta definición lleva implícita el adjetivo de universidad compleja y, a su vez, conduce a un cuerpo académico que debe poseer una masa crítica de investigadores; es decir, de generadores de conocimiento en sus respectivas áreas.

\subsubsection{Actividades y categorías académicas}

Un tercer elemento de esta nueva política está dado por las alternativas de carrera y de categorías académicas que se definen a partir de las actividades que pueden realizar los profesores. Estas son: 
- Docencia y formación de personas.

- Investigación y creación en alguna de sus formas.

- Extensión, difusión o aplicación del hacer propio de cada disciplina.

- Participación en la gestión de la universidad.

Debe enfatizarse el sentido amplio que se le ha dado a los términos "docencia" e "investigación":

- El concepto "docencia" se refiere no sólo al proceso enseñanzaaprendizaje, sino también a la formación de personas, entendida como una estructura de pensamiento o de acción en el quehacer propio de la disciplina y como formación ética y valórica necesaria para el ejercicio profesional.

- A su vez, el concepto "investigación" debe entenderse en un sentido amplio, abarcando la creación en diferentes ámbitos: intelectual, artístico, profesional, entre otros.

Bajo este marco, se pueden estructurar las categorías académicas que rigen al interior de la universidad. Para llevar adelante su misión, el cuerpo académico de la universidad estará conformado por dos categorías, "ordinaria y especial", cada una con objetivos y tareas específicas y complementarias. El núcleo del profesorado deberá estar preferentemente compuesto por docentes con alta dedicación y compromiso institucional, principalmente de categoría ordinaria. Este núcleo central se complementará con profesores en las categorías especiales, con diversas jornadas de dedicación y contratados para realizar actividades específicas.

a. Académico de categoría ordinaria:

Debe necesariamente, y como parte de su labor de base, realizar labores de docencia y de investigación o creación por sobre un cierto volumen mínimo, determinado al interior de cada unidad o facultad. A la luz de su plan de desarrollo y del perfil o características específicas del académico, cada facultad definirá el énfasis que éste pondrá en cada una de sus actividades, destacando que una docencia de calidad y la investigación o creación de alto nivel son irrenunciables para 
profesores de categoría ordinaria. Por lo tanto, un académico de esta categoría realiza docencia e investigación como actividad base y sobre ella construye su propio perfil de acuerdo con sus talentos e intereses propios, y las necesidades del plan de desarrollo de su unidad. Este perfil no debe entenderse como rígido, porque puede tener variantes a lo largo de la carrera del profesor.

b. Académico de categoría especial:

Esta categoría acoge a académicos que no necesariamente realizan ambas actividades de base (docencia e investigación), pudiendo incluso desarrollar sólo una de las cuatro actividades mencionadas. Una definición de este tipo no va en desmedro de la importancia del profesor para su unidad académica, ya que su actividad, más centrada en algunos aspectos, puede ser clave para el desarrollo y avance del plan estratégico respectivo.

\subsubsection{Carrera académica: base común y perfil académico diferenciado}

A la luz de lo expuesto en los párrafos anteriores, la PUC plantea la alternativa de diversificar las posibilidades de carrera académica, haciéndose cargo de las diferencias en aptitudes e intereses de sus académicos. Experiencias similares se encuentran descritas en literatura sobre algunas instituciones internacionales. Clark, en su libro "Creating Entrepreneurial Universities Organizational Pathways of Transformation", menciona la Universidad de Joensuu, en Finlandia, como un ejemplo de institución que arriesgó efectuar cambios significativos en su gestión y que logró con ello notables avances en cortos periodos. Allí se implementó, a partir de 1992, la llamada "carga académica flexible", a través de la cual los académicos acordaban con sus superiores, año a año, el porcentaje de dedicación de su jornada a actividades como docencia, investigación y extensión. Más recientemente, universidades como la de Melbourne han puesto en práctica nuevas políticas de gestión del cuerpo académico, que contemplan la posibilidad de carreras diferenciadas. Por su parte, la Universidad de Ottawa estableció el sistema principalmente en su Facultad de Medicina. La diferenciación de carreras académicas 
desplaza la exigencia de metas de calidad en docencia, investigación, extensión y gestión desde cada profesor, individualmente, a los grupos de trabajo en que se organizan las unidades académicas para realizar sus tareas. Esto implica que se reconozcan y establezcan ciertos grados de especificidad de funciones de acuerdo con la vocación y talento de cada uno.

De esta manera, se formalizarán carreras académicas individuales (perfiles) preferentemente dedicadas a la docencia o a la investigación, o a la extensión, o bien a la gestión y servicio universitario, dentro de equipos de trabajo que, como un todo, llevan adelante el Plan de Desarrollo, las metas y objetivos de la unidad académica a la cual pertenecen. El modelo descrito, basado en perfiles que enfatizan alguno de los ámbitos desarrollados por los académicos, de ninguna manera excluye la posibilidad de que uno de ellos, individualmente, desarrolle varias actividades, sin enfatizar particularmente en alguna.

Todo lo anterior no implica que éstos deban realizar actividades en cada uno de los ámbitos nombrados - "docencia, investigación o creación en alguna de sus formas, extensión y gestión”- y, menos aún, que puedan, razonablemente, exhibir un desempeño sobresaliente en todos. En consecuencia, la natural presencia de vocaciones, talentos y capacidades diversas para llevar adelante las distintas tareas hace recomendable fomentar el reconocimiento y cultivo de tal distinción de perfiles o características.

La asignación de actividades por cada académico en un periodo determinado, las que dan cuenta de su perfil, se materializan mediante convenios suscritos entre él y el decano, los que se constituyen en un antecedente del proceso formal de calificación. La aplicación de estos mecanismos es actualmente heterogénea en las distintas unidades universitarias, siendo uno de los objetivos de la nueva política favorecer su aplicación en todas las unidades. 
Por último, para una adecuada evaluación y calificación del desempeño académico individual, los profesores deberán definir, de común acuerdo con sus superiores jerárquicos inmediatos, la actividad que desarrollarán preferentemente -sea esta de tipo docente, de investigación o creación, de extensión o de gestión-, con el fin de que la programación de actividades de la unidad respectiva pueda armonizar el aporte de todos sus miembros.

\subsection{Política de Gestión del Cuerpo Académico: estrategias y mecanismos de implementación}

\subsubsection{Derechos y deberes}

Tanto entre los lineamientos que plantea la nueva Política de Gestión del Cuerpo Académico como en la normativa interna que deriva de ella y de otros cuerpos normativos de jerarquía mayor, se puede encontrar una serie de derechos y deberes. A continuación, se dan a conocer los de mayor relevancia.

La Política plantea que los profesores tienen derecho al ejercicio de la libertad académica, entendiendo por ella la posibilidad de analizar y exponer cualquier forma de pensamiento, pero aportando una apreciación objetiva y razonada.

Asimismo, tienen el derecho de reclamar ante la autoridad competente por cualquier acción u omisión que de alguna manera vulnere sus derechos reconocidos por los correspondientes cuerpos normativos. Ningún académico puede ser sancionado sino por acciones u omisiones que le sean imputables, y previo proceso formal de indagación.

Entre sus deberes podemos destacar el de la lealtad institucional, consagrado en la Declaración de Principios y Estatutos de la universidad. No tiene otras limitaciones que las impuestas por la moral, la legislación vigente y las normas de convivencia universitaria. 
También, es deber de todo académico contribuir a resguardar la unidad, la autonomía, el prestigio y el patrimonio de la universidad. Asimismo, la de evitar los conflictos de intereses que la afecten.

Por último, debe procurar siempre en sus actuaciones la debida solidaridad para con la comunidad universitaria de la que forma parte y, en especial, con las decisiones adoptadas por las autoridades competentes.

\subsubsection{Procesos de selección}

El proceso de selección de nuevos académicos debe ser riguroso, reflejando los altos estándares a los cuales quiere acceder la universidad en los próximos años. En todo caso, para ingresar a la Planta Académica Ordinaria el candidato debe tener como mínimo el grado académico de doctor o su equivalencia.

Por otra parte, una de las metas institucionales es lograr una mayor internacionalización, reflejada también en la composición de su cuerpo académico. Para nuestra universidad es un signo de fortaleza contar con profesores formados en diversos centros de excelencia, ya que ello demuestra tanto la atracción de talentos que esta institución tiene en el concierto internacional como el grado de apertura y riqueza que eso representa. Por este motivo, la institución promueve fuertemente que los concursos para proveer un nuevo cargo sean abiertos e internacionales, de manera de contar con candidatos altamente calificados de diversos centros nacionales e internacionales.

Lo anterior de ninguna manera excluye la posibilidad de incorporar, a través de concurso, académicos provenientes de nuestras propias aulas. En vez de dificultar su ingreso a la universidad, se propone que, de ser seleccionados, realicen estudios de perfeccionamiento de sus disciplinas en centros calificados. En el caso de haber obtenido el grado de doctor en la propia universidad, pueden completar su preparación con trabajos de posdoctorado en algún centro de referencia internacional. 


\subsubsection{Acogida, perfeccionamiento y desarrollo académico}

Un foco particular de la Política de Gestión del Cuerpo Académico lo constituyen las nuevas generaciones que se incorporan a la tarea universitaria, de manera que, después de un riguroso proceso de selección, puedan sentirse parte de la institución en un tiempo breve, logrando adecuados niveles de productividad.

Con este objetivo, la Dirección Superior ha puesto en práctica una serie de programas de apoyo a los académicos jóvenes: inserción institucional en docencia, fondos de inicio en la investigación, entre otros. También es fundamental el acompañamiento y orientación de las autoridades y profesores de mayor trayectoria de su unidad, quienes debieran considerar el apoyo a los más jóvenes como uno de los principales aportes que pueden realizar a sus respectivas comunidades académicas.

Cada unidad debe procurar que los nuevos ingresados, en sus dos o tres primeros años, se dediquen con preferencia a las actividades de investigación. Lo anterior no excluye algunas responsabilidades docentes y se dejarían para etapas más avanzadas de su carrera labores de gestión y extensión.

En concordancia con lo anterior, resulta necesario establecer programas de inducción y capacitación, orientados a la formación y perfeccionamiento del cuerpo académico, entregándole herramientas para su desarrollo personal, su comprensión del ámbito cultural, un ejercicio de la docencia del mejor nivel y para trasmitir a los alumnos conceptos y actitudes de solidaridad y responsabilidad social.

Sin duda, es importante considerar otros factores que inciden en la eficacia de este proceso, como aquellos de índole económica (recursos e incentivos), de infraestructura (calidad de los lugares de trabajo y equipamiento) y de vínculos internacionales (redes). 


\subsubsection{Evaluación y calificación}

Los académicos de la universidad, tanto de categoría ordinaria como especial, deben participar en evaluaciones bienales sobre la calidad de su trabajo. El resultado será considerado tanto para su promoción en la carrera académica como para la determinación de sus remuneraciones -como será descrito más adelante- y la asignación de funciones de responsabilidad individual en la facultad respectiva.

La evaluación tendrá en cuenta la calidad y cantidad de las actividades académicas realizadas, considerando su categoría, las funciones que le hayan sido asignadas y los acuerdos de desempeño alcanzados con las autoridades respectivas (perfil preferente). En la calificación global serán especialmente valoradas las actividades de colaboración con otros académicos, particularmente con los más jóvenes, las calificaciones y antecedentes anteriores. Se debe procurar que la continua expansión del conocimiento y la imbricación de intereses entre disciplinas diversas motive la creación de grupos de trabajo interdisciplinarios, cuyos ámbitos de investigación suelen abarcar límites epistemológicos tradicionalmente asignados a ciertas facultades y departamentos. Esto plantea la necesidad de renovar paradigmas históricos de organización, cuestión que marcará profundamente el futuro campo de acción de nuestros académicos.

Los criterios descritos para la calificación académica de cada profesor en función de su perfil y evaluando las tareas encomendadas se explicitan mediante las calificaciones: "Muy bueno", "Bueno", "Suficiente", "Con observaciones" e "Insuficiente". Haber obtenido la calificación de "Muy bueno" en al menos uno de los dos últimos periodos de calificación es una condición necesaria pero no suficiente para avanzar en su carrera. En un sentido contrario, la calificación "Insuficiente" o la reiteración de "Con observaciones" se entenderán como una recomendación para poner término a la relación contractual y de categorización del académico así calificado. 


\subsubsection{Cese de las labores académicas}

La PUC tiene la política del retiro obligatorio de los académicos a los 65 años, pero abre la posibilidad de que en ese momento las facultades, por acuerdo de sus respectivos consejos, puedan, por razones fundadas, prorrogar la permanencia de un profesor en la planta ordinaria hasta los setenta años, o a partir de ésta hasta por cinco años adicionales, conforme la aprobación de las instancias superiores pertinentes. A partir de este último caso, el académico puede ser postulado como "profesor emérito", sin mantener una relación contractual con la universidad.

También pueden seguir vinculados a la universidad aquellos académicos que, habiendo alcanzado la edad de retirarse de la planta, mantienen una actividad muy relevante para su facultad o para la universidad como un todo. Se actúa de esa manera porque se estima que, debido a sus cualidades personales, su trayectoria académica o porque es un modelo para las generaciones más jóvenes, su retiro sería una pérdida para la comunidad universitaria.

\subsubsection{Remuneraciones}

Parte de la gestión de unidades académicas depende de concordar y alinear elementos de una planificación, discutida y socializada entre las instancias más altas de la universidad y plasmada en su Plan de Desarrollo Institucional, con las propuestas de desarrollo de cada unidad académica, para conocer y sustentar un proyecto sinérgico común. De esta forma, los planes y propuestas de desarrollo de las unidades determinan proyecciones de tamaño de planta acordes con los niveles de actividad que ellos demandan y con las posibilidades de financiamiento sustentable.

En este esquema, la definición de una política de remuneraciones tiene un rol fundamental, en cuanto a la búsqueda de competitividad, atracción de talentos y transparencia de perspectivas de los profesores. Sin duda, esta definición redunda en lograr mayores grados de estabilidad en la conformación de los cuerpos académicos. Lo anterior se complementa con otros elementos fundamentales pero de un 
carácter más "intangible", como un ambiente favorable, sentido de pertenencia a la comunidad y reconocimiento y sello institucional, es decir, factores que condicionan el desempeño académico.

Para una constante retroalimentación de nuestra política de remuneraciones se debe considerar la diversidad de las facultades y las particularidades que ellas albergan. En este sentido, es necesario generar y monitorear información que favorezca la toma de decisiones sobre dicho ámbito, con el fin de tender a la búsqueda de la equidad interna y la competitividad externa, tanto con el ambiente universitario como con el mercado externo.

La política de remuneraciones y, en un sentido amplio, los criterios para la retribución de nuestros académicos se sustentan en cuatro componentes generales:

\subsubsection{Remuneración base y variable:}

Corresponde al principal grupo, ya que contiene los componentes que aseguran niveles mensuales relativamente constantes de ingresos y uniformes para la mayor cantidad de nuestros académicos.

a. Sueldo base (SB): determinado en función de la categoría que posee el profesor, conforme a una carrera académica definida, la cual incluye profesores asistentes, asociados y titulares en orden de menor a mayor trayectoria académica. El sueldo base es definido para cada categoría y, en conjunto con la dirección de las facultades, conforme a la información de realidad interna y externa recopilada.

b. Asignación de Desempeño Académico (ADA): es una componente variable en el tiempo, de menor cuantía, adicionada al sueldo base y cuya variación depende directamente del desempeño del profesor, medido en procesos bienales de calificación académica, situación descrita en el punto 2.2.4 de este documento. La metodología de vinculación a la remuneración de cada profesor es definida con cada unidad académica, haciendo que se incremente, mantenga o disminuya (esta última en circunstancias muy 
esporádicas) el componente variable de remuneración, según la calificación obtenida por cada profesor.

Figura 1: Estructura de Política de remuneraciones vinculada al desempeño

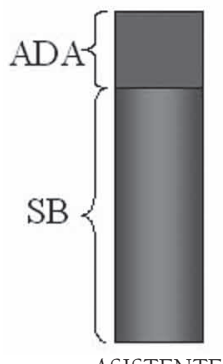

ASISTENTE

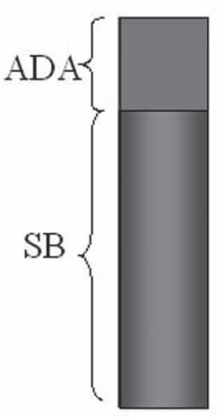

ASOCIADO

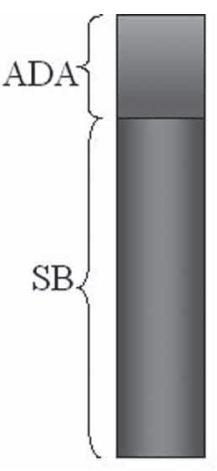

TITULAR

c. Asignación de Exclusividad Académica: asignación mensual complementaria al sueldo base, de mayor cuantía, que se revisa cada dos o tres años y que fue implementada en la década de los 90 con el fin de asegurar que los profesores no desarrollaran actividades fuera de la universidad. Su valor se diferencia según la categoría del profesor (ordinaria) y se adjudica a un porcentaje mayoritario de los profesores de mayor dedicación contractual. Esta práctica ha permitido incrementar el nivel de ingresos de nuestro núcleo académico, lo que ha redundado en una gran estabilidad de la planta. Los algoritmos de asignación son diversos, privilegiándose en algunas facultades la productividad, en otras la exclusividad o bien como una forma de reforzar a académicos que realizan funciones primordiales para la concreción de los planes de desarrollo de sus facultades.

d. Otros haberes: se enfocan a la retribución asociada a cargos de responsabilidad, servicio profesional y de extensión, entre otros. 


\subsubsection{Incentivos y premios:}

En nuestra universidad existen en este ámbito experiencias destacables, como los premios de investigación a la generación de publicaciones y a la excelencia en la docencia:

a. Premio a la Excelencia en la Docencia (PRED): aunque cuenta sólo con una primera versión, ha reconocido la excelencia de nuestros docentes según áreas disciplinares afines e incorporando en su metodología la elección de profesores jóvenes. Entre los criterios considerados para la determinación de una definición de calidad o excelencia en docencia encontramos: experticia actualizada en su área de conocimiento; impacto en logros de aprendizaje de los alumnos; metodologías de enseñanza y aprendizaje pertinentes al área de conocimiento; compromiso con la comunidad académica y con la realidad nacional, y cercanía del profesor a constituirse en un modelo para sus alumnos encarnando valores institucionales.

b. Programa de Reconocimiento a la Excelencia en Investigación (PREI): de larga data en nuestra universidad, incentiva y premia la publicación de artículos en revistas de corriente principal indexadas, como también libros y/o capítulos de libros. Los criterios de elegibilidad se basan en un proceso actualizado de forma permanente y que considera, entre otros criterios, el tipo de publicación (estrictamente investigación), la indexación de las revistas, los índices de impacto, productividad histórica reciente de los académicos, evaluadores, en el caso de libros y capítulos de libros. Cada año se determinan los montos asignados a cada académico en función de los recursos dispuestos para este programa y de los criterios antes descritos. Los premios adjudicados son de una gran cobertura entre nuestros investigadores, con montos variables para cada uno de ellos en función de la cantidad y calidad de sus publicaciones.

\subsubsection{Fondos concursables:}

En el último tiempo, y de forma creciente, ha surgido en el medio nacional una serie de programas de financiamiento a la investigación 
que disponen de diferentes mecanismos de asignación y control, conforme a las directrices definidas por cada organismo que convoca y adjudica los recursos. No existe una política única y consensuada con relación a la porción de fondos asignados como remuneración a los investigadores. A esto se agrega la realidad de otros recursos aportados por algunos organismos internacionales.

\section{Consideraciones finales}

Se espera que este documento de difusión, tanto de elementos conceptuales como de herramientas basadas en la experiencia de la PUC, en el contexto de definición de una Política para la Gestión del Cuerpo Académico "consensuada y discutida al interior de esta universidad", se constituya en un aporte significativo en la definición o revisión de otras experiencias universitarias en este ámbito.

Asimismo, se pretende que este marco impulse el desarrollo del cuerpo académico conforme a sus vocaciones y talentos, y que, junto con el desarrollo institucional y de las unidades, favorezca la evaluación de las actividades con el fin último de un aseguramiento constante y creciente de la calidad.

Agradecimientos: los autores agradecen a todos los que aportaron tiempo y esfuerzo a la estructuración tanto de la nueva Política de Gestión del Cuerpo Académico como del Reglamento del Académico que la lleva a la práctica; en particular, a los miembros de las comisiones ad-hoc nominadas para estos efectos. A su vez, se agradece a Karla Eliessetch, por su aporte en la redacción del presente documento.

\section{Referencias bibliográficas}

Clark, Burton R. (1998) Creating Entrepreneurial Universities Organizational Pathways of Transformation. IAU Press Pergamon, Issues in Higher Education, Second Edition.

Davis, Keith y Newstrom, John W. (2002) Comportamiento Humano en el Trabajo. México: McGraw-Hill. 
Koontz, Harold y Weihrich, Heinz. Administración: Una perspectiva global. México: McGraw-Hill, 2004.

Honorable Consejo Superior PUC (2008) Políticas de gestión del cuerpo académico en la Pontificia Universidad Católica de Chile. Santiago de Chile: Pontificia Universidad Católica de Chile.

Secretaría General. Vicerrectoría Académica PUC (2008) Reglamento del Académico. Santiago de Chile: Pontificia Universidad Católica de Chile.

PUC (2005) Plan de Desarrollo 2005-2010 "Duc in altum". Santiago de Chile: Pontificia Universidad Católica de Chile.

PUC (1982) Política académica. Santiago de Chile: Pontificia Universidad Católica de Chile.

Universidad de Melbourne, Australia. Personnel Policy and Procedures Manual. (Sitio en Internet) Disponible en: http://www.unimelb.edu.au/ppp/docs/

Universidad de Ottawa, Canadá. Policies. (Sitio en Internet) Disponible en: http://web5.uottawa.ca/admingov/policies.html

Recibido: 25 de abril de 2008

Aceptado: 27 de mayo de 2008 